\title{
Krisenhafte Lernprozesse im Entwicklungspolitischen Freiwilligendienst: Scheitern als Chance für Globales Lernen
}

\author{
Sonja Richter \\ Consultant I Globalcation - Research and Consulting on Global Education \\ sonja.richter@globalcation.de
}

\section{Zusammenfassung}

Dieser Beitrag stellt zentrale Ergebnisse einer qualitativ-empirischen Studie zur Qualität von Lernprozessen in entwicklungspolitischen Freiwilligendiensten vor. Der Fokus liegt hierbei auf nicht-intendierte Lernwirkungen, die konträr zu den jeweiligen Programmzielen im Sinne Globalen Lernens sind: Der als egoistisch betitelte Selbstbezug der Lernenden sowie die Verstärkung von rassistischen und stereotypischen Denkmustern gegenüber das Fremde. Die empirischen Befunde der Studie zeigen jedoch auf, dass diese negativ konnotierten Wirkungen einen wichtigen Zwischenschritt zu fundierten Lernprozesse Globalen Lernens, die in der Reflexionsphase nach der Programmteilnahme passieren, sein können.

Schlagwörter: Freiwilligendienst, Weltwärts, Globales Lernen, Lernprozesse

\begin{abstract}
This article presents results of a qualitative-empirical study on the quality of learning within volunteer services in the Global South. It focuses on unintended learning effects which do not go along with the respective program objectives like global learning: The self-orientation while experiencing difference and the reinforcement of racist and stereotypical patterns of thinking. However, the empirical findings of the study do show that these negative connotated effects can be an important and intermediate step towards a well-founded global learning process, which might occur after the service in the reflection phase.

Keywords: Volunteer Service, Weltwärts, Global Learning, Learning Processes
\end{abstract}

\section{Einführung: Lernen im entwicklungspolitischen Freiwilligendienst}

Die Frage des Lernens in entwicklungspolitischen Freiwilligendiensten steht in Deutschland spätestens seit der Überarbeitung der Förderlinie des Weltwärts-Programms im Zentrum der Diskussion um die Wirkung solcher Freiwilligendienstprogramme. Das ursprüngliche Motto des Programms „Lernen durch tatkräftiges Helfen“ (BMZ 2007: 4) wurde durch das Paradigma eines „Bildungsdienst[es]“ ersetzt, der „umfassende Möglichkeiten des Globalen Lernens eröffnet“ (BMZ 2014: 3). Dementsprechend soll Weltwärts Weltoffenheit und Toleranz fördern sowie zur Entwicklung von verantwortungsvollen Handlungskompetenzen in der globalen Welt beitragen. Die hierfür notwendige 
Reflexions- und Abstraktionskompetenz zeigt sich in den Evaluationen nur spärlich - es wird deutlich, dass die Freiwilligen überwiegend linear-kognitiv lernen und ein Verständnis von komplexen Zusammenhängen nur in Ansätzen auftaucht: Empirische Studien zu Lernen im Kontext von studien- und praxisbezogenen Auslandsaufenthalten im Globalen Süden deuten zwar darauf hin, dass Teilnehmende sich als interkulturell kompetent und weltoffener als vor dem Einsatz im Ausland einschätzen (z. B. ZEM 2010; Polak u. a. 2017). Fundierte Hinweise darauf, dass dieses Wissen auf andere fremdkulturelle Kontexte übertragbar ist, sucht man jedoch vergeblich, obgleich dies eine zentrale Zieldimension Globalen Lernens ist. Dementsprechend stellt die Programmevaluation „Weltwärts-Freiwillige und ihr Engagement in Deutschland“ fest, dass die Freiwilligen zwar Wissen über das Gastland oder eine Fremdsprache erwerben, dieses aber nicht auf andere Kontexte übertragen (Polak u. a. 2017: 62; 72 ff.).

In diesem Zusammenhang muss auch auf die Aussagekraft einiger Studien hingewiesen werden, welche aufgrund der evaluationsbasierten Methodik eingeschränkt ist. Vorrangiges Ziel von Programmevaluationsstudien ist es, die jeweiligen Programmziele zu überprüfen. Nicht intendierte Wirkungen, deren potenzielle Existenz außerhalb des gemeinen Vorstellungshorizont liegen, fallen hierbei unter den Tisch. Ein weiterer oft kritisierter Punkt ist die explizite Abfrage von „Lernergebnissen“ bei den Teilnehmenden, sodass das Antwortverhalten von sozialer Erwünschtheit geprägt ist.

Die Erkenntnisse der oben erwähnten Studien zeigen somit nicht auf, wie genau Lernprozesse im entwicklungspolitischen Freiwilligendienst „passieren“. Sie berücksichtigen weder die Bedeutung der Bildungsbiografie der Freiwilligen noch die Qualität des Einsatzplatzes. Der Charakter eines komplexen und in großen Teilen unbewussten Vorgangs wie der des Lernens kann in Evaluationen nicht vollständig abgebildet werden.

Dieser Beitrag knüpft genau hier an. Nachfolgend werden Ergebnisse einer qualitativ-empirischen Studie vorgestellt, in welcher unterschiedliche Qualitäten von Lernprozessen herausgearbeitet wurden. Es konnte aufgezeigt werden, welche Mechanismen individuelle Lernprozesse im Freiwilligendienst beeinflussen. Mit einem Einblick in die zentralen Ergebnisse dieser Studie und die Bedeutung dieser für die Konzeption von Freiwilligendienstprogrammen ergänzt dieser Beitrag auf den Output fokussierte Evaluationsergebnisse von Freiwilligendienstprogrammen. Im Fokus der Ausführungen stehen hierbei negativ konnotierte Lernphänomene, die üblicherweise nicht den Zielen der Programme entsprechen. Hierzu zählt unter anderem das „Erlernen“ von undifferenzierter Stereotypisierung, rassistischen Vorurteilen oder eine komplette Abwehr des Fremden. Dieser Beitrag zeigt auf, dass diese „negativen Lernergebnisse“ zwar im Rahmen des Programms (erstmal) passieren, aus einer biografisch-systemischen Perspektive 
heraus betrachtet jedoch die Grundlage für einen tiefgehenden „Prozess Globalen Lernens" ${ }^{\text {"1 }}$ sein können.

\section{Lernqualität verstehen: Eine empirische Studie zur Qualität von Lernen im Freiwilligendienst ${ }^{2}$}

Die empirische Grundlage der in diesem Beitrag vorgestellten Ergebnisse stellen Daten aus 22 narrativ-problemzentrierten Interviews (Schütze 1983; Witzel 1985) mit Rückkehrenden von Freiwilligen aus dem Globalen Norden sowie eine umfassende Kontextanalyse der organisationsbezogenen und individuellen Rahmenbedingungen dar. Ziel der Studie ist die Darstellung möglichst unterschiedlicher Qualitäten von Lernprozessen im entwicklungspolitischen Freiwilligendienst. Die interviewten ehemaligen Freiwilligen wurden dementsprechend gemäß dem theoretischen Sampling (Glaser/Strauss 1998) mit dem Ziel ausgewählt, ein möglichst diverses Datenmaterial zur Abbildung unterschiedlicher Lernqualitäten zu erhalten. Es umfasst ehemalige Teilnehmende nicht kommerzieller Entsendeprogramme mit dem Anspruch des „Lernens“ und gleichzeitigem Praxisbezug ab einer Aufenthaltsdauer von drei Monaten in einem Land des Globalen Südens. Die Zeitspanne zwischen Rückkehr und Interview (= Reflexionszeit der Erfahrungen) bewegt sich zwischen drei Monaten und zehn Jahren. Die Interviews wurden entsprechend dem narrativen Ansatz als offene Gespräche über Erfahrungen im Kontext der Programmteilnahme geführt, die Kontextanalyse zur Erfassung der Rahmenbedingungen stellt sich als Kombination aus einer Vorabumfrage der Interviewten und einer Desktopstudy dar.

Entsprechend dem qualitativen Forschungsparadigma erfolgte die Auswertung des Datenmaterials mit an das Erkenntnisinteresse angepassten Methoden: Im ersten Schritt wurde in einem hermeneutischen inhaltsanalytischen Verfahren herausgearbeitet, was die Teilnehmenden lernen, $o b$ bzw. wie sie das erlebte Fremde in den eigenen Orientierungshorizont integrieren sowie wann und in welchen Situationen diese Integration stattfindet oder nicht. Die Dateninterpretation wurde unter Einbezug der individuellen Bildungsbiografien sowie programm- bzw. organisationsbezogenen Rahmenbedingungen vorgenommen und kommunikativ validiert. Die Ergebnisse dieses Prozesses sind die Basis für den zweiten Auswertungsschritt: In einem abduktiv-zirkulären Prozess, der streng dem Vorgehen der empirisch begründeten Typenbildung nach Kluge (1999) folgte, wurde das Datenmaterial weiter abstrahiert und verdichtet.

1 Globales Lernen vermittelt über ganzheitliche Lernprozesse Bewusstsein und Handlungskompetenz für Individuen im Kontext von Globalisierung vor dem Leitbild globaler Gerechtigkeit.

2 Dieser Beitrag basiert auf Ergebnissen der empirischen Forschung des Dissertationsvorhabens „Zur Qualität von Lernen im Kontext von Freiwilligendiensten im Globalen Süden“ (Arbeitstitel) vor (Richter i.V.; Richter 2018) 
Als übergeordnetes Ergebnis zeigt sich, dass Lernen im Freiwilligendienst immer im Spannungsfeld von „Selbst“ (= die Persönlichkeit der lernenden Freiwilligen) und „Fremd“ (= das, was die Freiwilligen als subjektiv „fremd“ erleben) passiert. Die Positionierung des Selbst zum Fremden bestimmt hierbei die Qualität des Lernprozesses. Sie lässt sich an zwei Parametern festmachen: Der Lernorientierung (innen vs. außenorientiertes Lernen) sowie dem Weltverständnis (mono- vs. multiperspektivisches Lernverständnis). Die Lernorientierung zeigt sich in ihren Extremen als ein innenorientiertes Lernen, in welchem neue Erfahrungen vor allem mit Bezug auf das Selbst reflektiert werden und wirken. Beim außenorientierten Lernen hingegen erfolgt eine Kontextualisierung des Fremden (auch) im Außen; die Lernwirkung ist ebenfalls (auch) außenorientiert. Ein monoperspektivisches Weltverständnisses zeigt sich in einer einheitlichen Betrachtung des Fremden aus der eigenen Perspektive, die Vorstellung einer anderen Perspektive auf das Fremde existiert nicht, wohingegen ein multiperspektivisches Weltverständnis grundsätzlich davon ausgeht, dass andere Perspektiven auf das Erlebte vorhanden sind (dies impliziert jedoch nicht automatisch die Kompetenz, diese Perspektive einzunehmen).

Tabelle 1: Die Diversität von Lernprozessen im Kontext von Freiwilligendiensten im Globalen Süden

\begin{tabular}{|c|c|c|c|c|}
\hline & \multicolumn{2}{|c|}{ Lernorientierung } \\
\hline & & & $\begin{array}{c}\text { Innenorientiertes } \\
\text { Lernen }\end{array}$ & $\begin{array}{c}\text { Außenorientiertes } \\
\text { Lernen }\end{array}$ \\
\hline 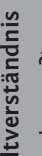 & 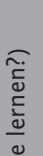 & $\begin{array}{l}\text { Monoperspektivisches } \\
\text { Weltverständnis }\end{array}$ & $\begin{array}{c}\text { TYP I } \\
\text { biografisch- } \\
\text { selbstbezogenes Lernen }\end{array}$ & $\begin{array}{c}\text { TYP II } \\
\text { biografisch- } \\
\text { karriereorientiertes Lernen }\end{array}$ \\
\hline 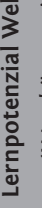 & 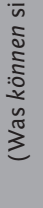 & $\begin{array}{l}\text { Multiperspektivisches } \\
\text { Weltverständnis }\end{array}$ & $\begin{array}{c}\text { TYP III } \\
\text { Hiatisches Lernen } \\
\text { (Lernen im Hiatus zwischen } \\
\text { Selbst und Fremd) }\end{array}$ & $\begin{array}{c}\text { TYP IV } \\
\text { Weltgesellschaftliches } \\
\text { Lernen („Globales Lernen“) }\end{array}$ \\
\hline
\end{tabular}

Quelle: Richter 2018: 18

Die Lernprozesstypen I und II repräsentieren Lernprozesse, in denen das „Selbst“ mit seiner eigenen Biografie gleichzeitig Ausgangspunkt und Ziel von Lernen ist. Die Integration des Fremden in das Selbst geschieht mit dem Ziel, die eigene Persönlichkeit mit Blick auf das Selbst (Typ I biografisch-selbstbezogenes Lernen) oder mit Orientierung auf die Berufslaufbahn (Typ II: biografisch-karriereorientiertes Lernen) zu entwickeln.

Mit Blick auf die Zielsetzungen entwicklungspolitischer Freiwilligendienste sind besonders Typ III und Typ IV interessant: das Lernen im Hiatus als Lernen, welches 
in der „Lücke“ zwischen Selbst und Fremd als Scheitern der Zielerreichung anzuordnen ist sowie Lernprozesse Globalen Lernens, welche für den Erwerb eines multiperspektivischen, systemischen Verständnisses von globalen Zusammenhängen und somit für eine positive Zielerreichung stehen.

Zwei übergreifende Ergebnisse der Studie werden jedoch auf den ersten Blick nicht mit den normativen Zieldimensionen Globalen Lernens in Verbindung gebracht: Der immer im Fokus stehende Selbstbezug, der in allen Lernprozessen entwicklungspolitischer Freiwilligendienste präsent ist und in seiner extremsten Ausprägung in Typ I, dem biografisch-selbstbezogenen Lernen, zeigt; sowie die Entwicklung von stereotypischen Haltungen auf das Fremde, die sich in rassistischen Äußerungen und Meinungen offenbaren. Letzteres Phänomen ist die nach außen sichtbare Wirkung von Lernprozesstyp III durch Überforderung mit dem Fremden und dem nicht überwindbaren Hiatus. Die Ausführungen in den folgenden zwei Kapiteln zeigen, dass diese im Hinblick auf die Programmziele „negativen" Phänomene dennoch Teil von Lernprozessen Globalen Lernens sind bzw. sein können.

\section{Das Selbst: Ursprung und Ziel von Lernprozessen im entwicklungspolitischen Freiwilligendienst}

Vor über zehn Jahren hat Tobias Töpfl in der Süddeutschen Zeitung als einer der ersten außerhalb der Freiwilligendienstszene das damals gerade ins Leben gerufene Programm Weltwärts scharf kritisiert: Unter dem vielzitierten Titel „Egotrip ins Elend" proklamierte er, dass Weltwärts vor allem den Freiwilligen selbst helfen würde und nicht den Menschen an den Einsatzorten (Töpfl 2008). Die Kritik wurde vielfach aufgenommen (z. B. Bandura 2018; Kontzi 2011) und bezog sich insbesondere auf die erste Programmkonzeption, in der das Entwicklungsparadigma im Vordergrund des Aufenthaltes stand und „Helfen vor Ort“ als zentrale Motivation bei den Teilnehmenden verankert war (BMZ 2007). Der Kritikwelle folgte ein Aufschrei in der Freiwilligendienstszene, der als eine Mischung aus Abwehr, Rechtfertigung und Selbstkritik beschrieben werden kann und schließlich in die programmatische Neukonzeption von Weltwärts als Bildungsprogramm mündete (BMZ 2014; 2016).

Das „Ego“ in Töpfls Headline kann auf das „Selbst“ der Freiwilligen bezogen werden, denn: Es steht letztendlich für nichts anderes als die individuelle Persönlichkeit der Freiwilligen; für deren individuellen Werte und Einstellungen und somit auch für bestimmte Verhaltensweisen dieser. Das Ego ist somit gleichzeitig auch ein zentraler Ansatzpunkt für die Entwicklung eines bestimmten Bewusstseins, Verhaltens oder Handelns - und genau das wollen entwicklungspolitische Freiwilligendienste bei den Teilnehmenden ja beeinflussen. 
In der empirischen Analyse der hier beschriebenen Forschungsarbeit zeigt sich tatsächlich, dass der Selbstbezug ein zentrales Merkmal von Lernen im Freiwilligendienst ist. Bei allen Fällen im Sample ist das Selbst eine wichtige Bezugsgröße, bei vielen sogar die dominante (vgl. Lerntyp I und II). Das erlebte Fremde wird instrumentalisiert und dient als Spiegelbild zur Reflexion und Entwicklung des Selbst. In der Auswertung der Daten zeigt sich, dass die Voraussetzung für Lernprozesse, in welchen das Fremde auf der Inhaltsebene im Lerngeschehen zum Tragen kommt (und nicht als Medium), ein multiperspektivisches Weltverständnis ist. Im Gegensatz zu den Freiwilligen mit dominantem Selbstbezug zeigt sich bei Freiwilligen mit multiperspektivischen Weltverständnis, dass (1) das Fremde in seiner Qualität sowie (2) die Differenz zwischen dem Selbst und dem Fremden jeweils wahrgenommen werden. Das Potenzial von Lernen durch eine Überwindung von Differenz wird erkannt, das Fremde ist nicht nur Medium, sondern Gegenstand des Lernprozesses.

Eine im Sinne Globalen Lernens erfolgreiche Überwindung dieser Differenz zeigt sich im Lernprozesstyp IV „Globales Lernen“: Das Fremde verändert das Selbst auf konstruktive Art und Weise, es hat Einfluss auf Einstellungen und Verhalten. Im hiatischen Lernprozess (Lernprozesstyp III) hat das Individuum zwar genau diesen Anspruch - wird diesem aber nicht gerecht. Das Scheitern am Hiatus - an der Differenz zwischen Selbst und Fremd - führt zwar zu einer Wahrnehmung des Fremden als Gegenstand, dieser wird jedoch schemenhaft wahrgenommen und negativ konnotiert. Hinsichtlich der Lernwirkung bedeutet dies oft, dass die Freiwilligen das erlebte Fremde stereotypisieren und pauschalisieren.

Auffallend ist, dass die anvisierten Lernprozesse Globalen Lernens sich bei Lernenden mit verhältnismäßig gefestigter Persönlichkeit zeigen. Freiwillige, die in der Lage sind, das Fremde differenziert und wertfrei zu betrachten, um dann eine Brücke zwischen Selbst und Fremd zur Überwindung der Differenz zu schlagen, greifen auf eine längere Lebenserfahrung zurück. Insbesondere vorangegangene Auslandsaufenthalte und praxisbezogene Erfahrungen spielen hierbei eine bedeutende Rolle. Angehende Weltwärts-Freiwillige können in der Regel auf einen solchen Erfahrungsschatz nicht aufbauen - die Sammlung jener ist ja genau ein Zweck des Programms.

Der entwicklungspolitische Freiwilligendienst Weltwärts ist ein Instrument deutscher Entwicklungspolitik - staatlich gefördert und zivilgesellschaftlich umgesetzt. In der Historie ist das Programm somit bei der vermeintlich altruistischen „Entwicklungshilfe“ verortet. Die Erkenntnis, das Programm fördere „Egotrips“, ist mit diesem Verständnis schwer vereinbar.

Rekonstruiert man Lernerfahrungen im Kontext der Freiwilligendienste jedoch unter Einbezug der jeweiligen (bildungs-)biografischen Lebensläufe, zeigt sich 
ein anderes Bild: Dann dienen genau diese selbstbezogenen Lernerfahrungen als Bezugsquelle für die Einordnung von vergleichbaren oder konträren Erfahrungen weit ab des Freiwilligendienstkontextes. Die selbstorientierte Einordnung des Fremden kann zu einem späteren Zeitpunkt reflektiert und zum Ausgangspunkt für multiperspektivische, globalorientierte Lernprozesse werden.

Innenschau und Selbstreflexion sind die Basis für Veränderungen im Außen, im Handeln und Verhalten. Dieser Ansatz wird auch in therapeutischen und persönlichkeitsentwickelnden Beratungsprozessen genutzt. Vor dem Hintergrund der Zielsetzung, Individuen zu differenzierenden, reflektierten Menschen mit einem Bewusstsein über globale Zusammenhänge ausbilden zu wollen, können Erfahrungen im Kontext eines Freiwilligendienstes im Globalen Süden genau hierfür die Grundlage sein.

\section{Stereotypisierung und Hierarchisierung: Die Überforderung mit dem Fremden als Teil des Globalen Lernprozesses}

Entwicklungspolitische Freiwilligendienstprogramme stehen in der Kritik, postkoloniale Strukturen und Machtungleichheiten zu verstärken. Im Rahmen der Programmteilnahme würden die Freiwilligen Interkulturalität nicht differenziert betrachten lernen, sondern durch eine „Strategie des Othering“ (Kontzi 2015: 165 ff., in Anlehnung an Said 1978) das erlebte Fremde aus einer Überlegenheitshaltung heraus stereotypisieren und pauschalisieren. Othering steht für Zuschreibungen der Freiwilligen auf das Fremde und verstärkt aufgrund der bereits vorhandenen Machtungleichheiten die Hierarchisierung zwischen Nord und Süd.

Das Phänomen des Othering zeigt sich auch in der hier beschriebenen empirischen Forschung - in den sogenannten hiatischen Lernprozessen (Lernprozesstyp III). Die Freiwilligen scheitern am Umgang mit der Differenz zwischen Selbst und Fremd - genauer gesagt am Anspruch der konstruktiven Integration des Fremden in das Selbst. Ihr Umgang mit der Differenz zeigt sich dann über eine Schematisierung des Fremden anhand einzelner Erfahrungen (Pauschalisierung und Stereotypisierung), die von einer hierarchischen Positionierung des Selbst über das Fremde geprägt ist.

Dass diese Prozesse tatsächlich stattfinden, zeigt sich im empirischen Material der hier zugrundeliegenden Studie. Die Bedeutung des Phänomens für Lernprozesse, die über den Freiwilligendienst hinaus gehen, legt eine starke Dichotomisierung zwischen dem Selbst und dem „Anderen“ offen. Ebenso werden auf der Mikroebene Machtungleichheiten durch Hierarchisierung des Selbst über das Fremde reproduziert. Besonders problematisch wird dieses Phänomen vor allem dann, wenn Freiwillige mit diesem Mindset der Programmempfehlung von Weltwärts folgen und im weiteren Sinne in der entwicklungspolitischen Bildungsarbeit aktiv 
werden, indem sie zum Beispiel über ihre Erfahrungen berichten oder in der Vorbereitung anderer Teilnehmende mitwirken.

Die empirische Analyse legt aber auch offen, dass der pauschalisierende und hierarchisierende Umgang mit dem Fremden auch für die Freiwilligen selbst als unbefriedigend empfunden wird und dass genau dieses dekonstruktive Handlungsmuster in Kombination mit dem negativen Gefühl des Scheiterns Ausgangspunkt für weitere, positiv konnotierte Lernprozesse in Richtung Globalen Lernens sind.

Stereotypisierung und Hierarchisierung zeigt sich fast immer in Kombination mit Scheitern und Frust, oft auch nur implizit. Die analysierten Interviews sind geprägt von Negativität, nicht nur gegenüber dem Fremden, sondern auch gegenüber sich selbst. Das Gefühl, es „,nicht geschafft“ zu haben, den Programmerwartungen nicht gerecht zu werden, frustriert die Freiwilligen (explizit und implizit), denn schließlich haben sie auch an sich den Anspruch, interkulturell kompetent und weltoffen zu werden.

Bei genauer Datenanalyse wird deutlich, dass die Freiwilligen das Fremde nicht in böser Absicht mit einem Überlegenheitsdenken pauschalisieren. Die bildungsbiografischen Hintergründe machen einen differenzierten Blick auf das Fremde im Freiwilligendienst aus entwicklungspädagogischer Sicht häufig schlichtweg unmöglich. Fehlende Möglichkeiten, die eigene Reflexionskompetenz vor der Programmteilnahme entsprechend zu entwickeln, führen dazu, dass die Freiwilligen nur schwer Anknüpfungspunkte zwischen dem eigenen Selbst und dem Fremden herstellen können. Es zeigt sich sogar, dass bei Teilnehmenden mit wenig praxisbezogener oder interkultureller Vorerfahrung ein konzeptionell vorgegebenes Bildungsziel hinderlich für ein positiv konnotiertes Lernerlebnis im Freiwilligendienst sein kann! Die Freiwilligen versteifen sich zu sehr auf den Anspruch, „positiv zu lernen“ - wenn dies nicht gelingt, ist die Frustration umso größer.

Lernprozesse Globalen Lernens zeigen sich nur in Facetten und auch nur bei den Teilnehmenden, die bereits auf Vorerfahrungen und somit auf eine gewisse Differenzierungs- und Reflexionskompetenz zurückgreifen können.

Betrachtet man die Analyseergebnisse mit Fokus auf den Lernprozess nach der Rückkehr, wird allerdings deutlich: In den Fällen, in denen die Teilnehmenden zum Zeitpunkt des Interviews die eigene Stereotypisierung kurz nach dem Dienst reflektieren können, hat ein ganz tief gehender, fundierter Lernprozess stattgefunden: Die ehemaligen Freiwilligen reflektieren ihr Verhalten, ihr „Scheitern“ im Umgang mit dem Fremden. Sie weisen dann eine Differenziertheit in der Betrachtung des Fremden auf, die aus ihnen selbst (und nicht, weil sie es in den Vorbereitungsseminaren vermeintlich gelernt haben) herauskommt. Das Scheitern, die Entwicklung einer stereotypischen Einstellung, kann somit Teil eines ganz 
fundierten Lernprozesses sein, wenn die entsprechenden Ankerpunkte zur Reflexion dessen nach der Rückkehr vorhanden sind.

\section{Fazit}

Eine ausschließliche Fokussierung auf das Selbst, die Entwicklung von Stereotypen und das Othering offenbaren sich als nicht intendierte Lernwirkungen im entwicklungspolitischen Freiwilligendienst. Die hier ausgeführten empirischen Ergebnisse legen jedoch auch dar, dass diese im Hinblick auf die Programmziele negativ konnotierten Lernprozesse Ausgangspunkt für Lernen im Sinne Globalen Lernens sein können. Es konnte aufgezeigt werden, dass diese „negativen Wirkungen" in einem dem Auslandsaufenthalt nachgelagerten Prozess nicht nur überwunden, sondern Ausgangspunkt eines viel tiefer gehenden Lernprozesses sind, der aus den Teilnehmenden selbst herauskommt. Die ehemaligen Freiwilligen können so erfahrungsorientiert lernen, das Fremde differenziert betrachten und die eigene Rolle in globalen Machtverhältnissen reflektieren. Die Relevanz des Selbstbezuges in Lernprozessen zeigt, dass neben organisationalen Rahmenbedingungen vor allem individuelle Bildungsbiografien, die das Selbst und somit die Lernprozesse charakterisieren, von großer Bedeutung für das Lernen im Freiwilligendienst sind. Denn nur über eine Anknüpfung an individuelle Vorerfahrungen, an frühere Lernerfahrungen der Freiwilligen ist es möglich, die Differenz zwischen Selbst und Fremd zu überwinden und das „Andere“ aus einer Multiperspektivität heraus differenziert wahrzunehmen.

Für die Konzeption und Umsetzung von Freiwilligendienstformaten in Politik und Praxis bedeuten die hier dargelegten Erkenntnisse zum einen, dass die Zielsetzungen der Förderprogramme entwicklungspolitischer Freiwilligendienste zu ambitioniert formuliert sind. Eine „Verwandlung“ von jungen Menschen mit wenig Lebenserfahrung zu reflektierten, differenzierten Multiplikator*innen für entwicklungspolitische Themen kann ein Freiwilligendienst alleine nicht leisten. Die Teilnahme an einem entsprechenden Programm kann jedoch zu einem Wissenszuwachs führen sowie Reflexionsprozesse anstoßen oder Engagement verstärken. Die Studie unterstreicht, dass Lernen ein komplexer Prozess ist, in welchem Erfahrungen im Kontext eines entwicklungspolitischen Freiwilligendienstes im Globalen Süden vor dem Hintergrund des eigenen Reflexionsrahmens verarbeitet wird. Die besondere Differenz zwischen fremden Erfahrungen und dem Selbst bedingt, dass Lernen auf der Grundlage dieser Erfahrungen in der Regel über mehrere Jahre oder Jahrzehnte andauert.

Für die Umsetzung entsprechender Programme empfiehlt es sich unter anderem, die hochgesetzten Erwartungen hinsichtlich des Lernprozesses Typ IV zu überprüfen. Die pädagogische Begleitung sollte auch Scheitern und Frust sowie stereotypisierende Verhaltensweisen erlauben und die Freiwilligen bei der Reflektion 
dieser individuell unterstützen. Negative Erfahrungserlebnisse im Rahmen der Programmteilnahme sind normal und sollten insbesondere in den Nachbereitungsseminaren wertfrei aufgenommen und adressiert werden. Wünschenswert wäre es, wenn die Nachbereitung Räume eröffnet, in welchen die Freiwilligen beginnen, ihr Scheitern, ihre Haltung zu reflektieren und somit das Ende des Freiwilligendienstes als Beginn (und nicht den Abschluss) eines tiefer gehenden Lernprozess verstehen.

\section{Literaturverzeichnis}

Bandura, Leander (2018): Wer entwickelt hier wen?, in: Der Freitag, online-Ausgabe: www.freitag.de/autoren/lfb/wer-entwickelt-hier-wen (01.05.2019).

BMZ - Bundesministerium für wirtschaftliche Entwicklung und Zusammenarbeit (2007): Förderleitlinie zur Umsetzung des entwicklungspolitischen Freiwilligendienst „weltwärts“, https://www.weltwaerts.de/en/detail.html?id=64\&file=files/_media/content/ Dokumente/3_EO/Infomaterial-EO/Foerderleitlinie-weltwaerts.pdf (27.03.2019).

BMZ - Bundesministerium für wirtschaftliche Entwicklung und Zusammenarbeit (2014): Förderleitlinie zur Umsetzung des entwicklungspolitischen Freiwilligendienstes weltwärts, Bonn.

Kontzi, Kristina (2011): Ich helfe, du hilfst, ... ihnen wird geholfen. Der Freiwilligendienst weltwärts reproduziert altbekannte Strukturen, in: Informationszentrum Dritte Welt, Heft 323, S. 40-42.

Kontzi, Kristina (2015): Postkoloniale Perspektiven auf „weltwärts“. Ein Freiwilligendienst in weltbürgerlicher Absicht. Zugl.: Lüneburg, Univ., Diss., 2014. 1. Aufl. Baden-Baden.

Polak, Jan Tobias, Kerstin Guffler und Laura Scheinert (2018): weltwärts-Freiwillige und ihr Engagement in Deutschland, hrsg. von DEval - Deutsches Evaluierungsinstitut der Entwicklungszusammenarbeit, Bonn.

Richter, Sonja (2018): Lernen zwischen Selbst und Fremd. Zur Qualität von Lernprozessen in Freiwilligendiensten im Globalen Süden, in: ZEP 1/2018, S. 17-22.

Richter, Sonja (in Vorbereitung): Zur Qualität von Lernen im Kontext von Freiwilligendiensten im Globalen Süden, Dissertationsvorhaben.

Schütze, Fritz (1983): Biographieforschung und narratives Interview, in: Neue Praxis 13. Jg. (3), S. 283-293.

Töpfl, Florian (2008): Egotrip ins Elend, in: Süddeutsche Zeitung Magazin, 08.05.2008, http://sz-magazin.sueddeutsche.de/texte/anzeigen/24384 (01.04.2010).

ZEM - Zentrum für Evaluation und Methoden (2010): Abschlussbericht. Unabhängige Evaluierung des ASA-Programms - beauftragt von InWEnt - Internationale Weiterbildung und Entwicklung gGmbH, Bonn. 